This is the submitted version of the article:

Esplandiu M.J., Reguera D., Fraxedas J.. Electrophoretic origin of long-range repulsion of colloids near water/Nafion interfaces. Soft Matter, (2020). 16. : 3717 - . 10.1039/d0sm00170h.

Available at: https://dx.doi.org/10.1039/d0sm00170h 


\section{Electrophoretic Origin of Long-range Repulsion of Colloids near Water/Nafion Interfaces}

Maria J. Esplandiu ${ }^{\dagger *}$, David Reguera ${ }^{\$ *}$, Jordi Fraxedas $^{\dagger *}$

${ }^{\dagger}$ Catalan Institute of Nanoscience and Nanotechnology (ICN2), CSIC and BIST, Campus UAB, Bellaterra, 08193 Barcelona, Spain

${ }^{\S}$ Departament de Física de la Matèria Condensada, Universitat de Barcelona, C/Martí i Franquès 1, 08028 Barcelona, Spain

FUniversitat de Barcelona, Institute of Complex Systems (UBICS), C/Martí i Franquès 1, 08028

Barcelona, Spain.

AUTHOR INFORMATION

Corresponding Author

*mariajose.esplandiu@icn2.cat, jordi.fraxedas@icn2.cat 


\begin{abstract}
One of the most striking properties of Nafion is the formation of a long-range solute exclusion zone (EZ) in contact with water. The mechanism of formation of this EZ has been the subject of a controversial and long-standing debate. Previous studies by Schurr et al. and Florea et al. root the explanation of this phenomenon in the ion-exchange properties of Nafion, which generates ion diffusion and ion gradients that drive the repulsion of solutes by diffusiophoresis. Here we have evaluated separately the electrophoretic and chemiphoretic contributions to multi-ionic diffusiophoresis using differently charged colloidal tracers as solutes to identify better their contribution in the EZ formation. Our experimental results, which are also supported by numerical simulations, show that the electric field, built up due to the unequal diffusion coefficients of the exchanged ions, is the dominant parameter behind such interfacial phenomenon in presence of alkali metal chlorides. The EZ formation depends on the interplay of the electric field with the zeta potential of the solute and can be additionally modulated by changing ion diffusion coefficients or adding salts. As a consequence, we show that not all solutes can be expelled from the Nafion interface and hence the EZ is not always formed. This study thus provides a more detailed description of the origin and dynamics of this phenomenon and opens the door to the rational use of this active interface for many potential applications.
\end{abstract}

Keywords. Nafion, exclusion zone, diffusiophoresis, electrophoresis, ion-exchange materials 


\section{Introduction}

Many materials build-up a large exclusion zone (EZ) to solutes when immersed in an aqueous solution. EZs have been observed in the vicinity of biological tissues ${ }^{1}$, white blood cells ${ }^{2}$, some metals $^{3}$, hydrogels $s^{4}$ and ion exchange polymers ${ }^{5}$. One of such polymers is Nafion, which is a copolymer with a comb-like structure made of perfluorovinyl ether groups terminated with sulfonic acid groups onto a tetrafluoroethylene backbone ${ }^{6,7,8}$. EZs of more than $200 \mu \mathrm{m}$ have been observed at Nafion/water interfaces when using colloidal tracers acting as solutes ${ }^{9,10,11}$. Such solute-free region cannot be ascribed to the negative polymer surface charge since its pure electrostatic influence only reaches a narrow region near the interface (below $1 \mu \mathrm{m}$ ) where the double layer is built-up in presence of water.

Important efforts have been invested in order to understand such peculiar phenomenon during the last years. Pollack and coworkers have attributed the EZ formation to water structuring which prevents the incorporation of particles or solutes in the water network ${ }^{9,10,12,13,14,15}$. That is surprising since, in other materials which are well known for inducing water ordering, the structured water layer extends only a few monolayers (few nanometers) from the interface ${ }^{16,17}$. The same research group also observed that the EZ induces a pH gradient and therefore charge separation ${ }^{9}$. Alternatively, Schurr and coworkers opened a debate by rooting the formation of EZ in chemotaxis driven by solute gradients $\left(\mathrm{OH}^{-}, \mathrm{H}^{+} \text {and salts in solution }\right)^{18,19}$. Such gradients would be large enough for displacing microparticles tracers which would move in favor or against such gradient depending on their surface functional groups (basic or acidic ones) and the interaction of these groups with the ions present in solution. In line with such hypothesis, the group of Wyss suggested that EZ formation is caused by a combination of ion-exchange at the 
surface, diffusion of ions, and diffusiophoresis of particles in the resulting ionic gradients ${ }^{11,20}$. Ion-exchange takes place at the active interface, where cations present in the solution are exchanged for $\mathrm{H}^{+}$ions of higher mobility. Such exchange sets up a gradient of ions, which in turn causes the colloidal particles in the suspension to move away from the active surface via diffusiophoresis. Under the light of the recent results, it has been established that the formation of an EZ in Nafion is due to ionic diffusiophoresis whose fundamental theoretical framework has been previously well settled ${ }^{21,22,23,24,25}$. Thus, the exclusion zone formation in Nafion is not a unique phenomenon, but rather shares similar roots with many interesting phoretic phenomena acting on colloids, macromolecules or biological entities such as particle banding ${ }^{25}$, particle focusing $^{26}$, particle/macromolecule patterning ${ }^{27,28}$ or bacterial chemotaxis ${ }^{29}$, among others. Moreover, and under similar context, very interesting particle self-assembly and exclusion zone formation have been also observed with ion-exchange resins acting as modular micro-swimmers in presence of passive colloidal particles on charged substrates ${ }^{30,31}$.

Given the controversies that persist in the interpretation of the EZ formation in Nafion films and other hydrophilic ion-exchange surfaces, it is worth analyzing the Nafion interface in further detail and investigating which are the key physicochemical parameters that modulate the EZ formation under different conditions. A good understanding of the Nafion interface is very important to improve the levels of control of their already well known or future envisioned applications. For instance, Nafion is a widely used material both in fuel cell technology and in the chlor-alkali industry due to its ion-exchange and ion conduction capabilities ${ }^{6,7,32,33}$. It is also a popular material in the fabrication of biosensors due to its biocompatibility, excellent thermal stability, mechanical strength and antifouling properties ${ }^{34,35}$. Moreover, a deeper knowledge of the EZ formation and how to modulate it could lead to exploit new phenomena such as the 
generation of self-sustained motion of fluids at interfaces by ion gradients with novel applications in advanced antifouling coatings ${ }^{36,37}$, microfluidics ${ }^{38,39}$, actuators and self-propelled motors $^{30,31,40,41,42,43,44,45,46,47}$.

The theoretical work by Schurr et al. ${ }^{18,19}$ and the experimental evidences by Florea et al. ${ }^{11}$ have placed the focus on diffusiophoretic forces on solutes as the main cause for interfacial solute rejection. In the previous work by Florea et al. the velocity of the colloidal particles moving away from the Nafion interface was considered proportional to the gradient of the logarithm of the total ionic concentration, being the proportionality parameter a global diffusiophoretic coefficient which was dependent on the Debye length, zeta potential, viscosity, temperature and diffusion coefficients of the ions. Such formulation, which is perfectly valid for dilute binary electrolytes, does not single out the electrophoretic and chemiphoretic contributions of multiionic diffusiophoresis, and becomes inaccurate to capture the dynamics of ion transport of multiple electrolytes as discussed for instance by Donev et al. ${ }^{48}$ and Gupta et al. ${ }^{49}$

In this work, we have tried to provide a more detailed picture of the EZ formation, by assessing experimentally the electrophoretic and chemiphoretic contributions of the diffusiophoretic forces on colloidal tracers at thin Nafion supported films in presence of alkali metal chlorides. We have evaluated the electric field and electrophoretic contribution of multiionic diffusiophoresis which arises as a consequence of the unequal diffusion coefficients of the exchanged monovalent ions. We have identified the electrophoretic forces as the main contribution involved in the EZ formation at such thin Nafion films. We show that not all solutes can be expelled from the Nafion interface and hence an EZ is not always formed. The EZ formation mainly depends on the electric field strength generated by ion exchange/diffusion and on the solute's zeta potential. Thus, the electrophoretic term is in this case the dominant 
contribution dictating the approach or rejection of the particle co-solute at the polymer interface and accounts for a more detailed scenario of the origin and dynamics of the EZ.

\section{Experimental}

\subsection{Sample preparation}

Nafion source was a commercial Nafion dispersion in the protonated form (Aldrich, $10 \%$ in water, eq. weight 1,100 ) without further purification. Nafion thin layers were prepared by spinning a diluted Nafion dispersion (5\%) in isopropanol either on $\mathrm{Au} / \mathrm{Si}$ or on $\mathrm{Si}$ wafers followed by a heating step at $100{ }^{\circ} \mathrm{C}$ for 5 minutes. The spinning process was repeated to achieve typical thicknesses of about $600 \mathrm{~nm}$ as measured by atomic force microscopy. Although $\mathrm{Si}$ supports were used for characterization of Nafion thickness and roughness, we found that the adhesion of Nafion to Au is much larger than in the case of Si supports. Hence most studies were finally performed on Nafion on Au substrates. For a better focalization of the Nafion surface with an optical microscope the gold support was patterned with $50 \mathrm{~nm}$ thick chromium marks previous to Nafion deposition. Moreover, for improving the first glance visualization of the EZ formation, the Nafion surface was also microstructured by patterning 500 and $100 \mu \mathrm{m}$ wide strips with e-beam lithography, respectively. The e-beam lithography modifies the Nafion composition by the scission of $-\mathrm{SO}_{3}{ }^{-}$moieties which yield to the loss of its ion-exchange capabilities (See Fig. S1).

Before the inspection of EZ, the Nafion surface was pre-moistened in Milli-Q water for 30 minutes. Contact angle measurements show that the Nafion wettability changes from a hydrophobic contact angle of $103^{\circ}$ to a hydrophilic contact angle of $20^{\circ}$ over time which is due to water uptake and surface compositional restructuration of Nafion in contact with water (Fig. S2). 
The Nafion pre-wetting helps to protonate the sulfonate moieties and to minimize the fluid motion due to water uptake when inspecting the built-up of EZ. We also evaluated the ion exchange capacity of such thin films which turned out to be $4 \times 10^{-3}$ moles of $\mathrm{H}^{+} / \mathrm{cm}^{3}$ in agreement with the literature ${ }^{11}$. The ion exchange capacity was measured by using confocal fluorescence with fluorescent $\mathrm{pH}$ indicators as detailed in the supplementary data (Fig. S3).

\subsection{EZ characterization}

Microparticles with $2 \mu \mathrm{m}$ diameter of different charges were used as tracers to visualize the EZ: microbeads of both plain and carboxylated PS spheres (Kisker Biotech GmbH \& Co) and PS spheres functionalized with amidine groups (Invitrogen). The concentration of particles was $0.2 \mathrm{mg} / \mathrm{ml}$ (volume fraction $=1.9 \times 10^{-4}$ ) and were dispersed in different concentrations of salt from $1.2 \times 10^{-6}$ to $1.0 \times 10^{-3} \mathrm{M}$. The values of zeta potential $(\zeta)$ of the particles used for estimating the electric field were $-0.012 \pm 0.001 \mathrm{~V},-0.042 \pm 0.003 \mathrm{~V}$ and $+0.046 \pm 0.002 \mathrm{~V}$ for the plain PS, carboxylate and amidine modified tracers, respectively. The $\zeta$ values were obtained for the particles dispersed in a salt concentration of $1.2 \times 10^{-6} \mathrm{M}$ with a Malvern ZetaSizer Nano ZS Instrument and using the Helmholtz-Smoluchowski equation as an approximation since the relation $r / \lambda$ between the particle radius (r) and the Debye length $(\lambda)$ was $r / \lambda=3.6$ and not strictly $r / \lambda>>1$ as required for the Smoluchowski limit. This approximation was nevertheless used since a pure experimental analysis taking into account the concentration dependence of the zeta potential would have not been feasible in the presence of an ionic concentration gradient. The average $\mathrm{pH}$ and conductivity of the tracer dispersions were $6.6 \pm 0.1$ and $1.15 \pm 0.01 \mu \mathrm{S}$, respectively. An average residual salt concentration at the particle dispersion of $1.24 \pm 0.06 \mu \mathrm{M}$ was measured by performing calibration curves of conductimetry under $\mathrm{CO}_{2}$ 
degassed conditions. The residual salts come from the synthesis process of the particles. $\mathrm{NaCl}$ or $\mathrm{KCl}$ salts are expected to be the more common ones since such alkali metal cations and chlorides are typical species involved in the polymerization and functionalization reactions of such colloidal particles. Such amount of salt is enough to trigger the ion exchange with the pristine proton loaded Nafion film.

In order to observe and quantify the EZ formation two different approaches were used. A qualitative observation was easily achieved by inspecting a Nafion-coated gold surface which was strip-patterned with electron beam lithography. These samples, previously soaked in MilliQ water, were then immersed in the colloidal dispersion and inspected with an inverted optical microscope with the Nafion sample on top and the objectives below (see Scheme in the supplementary data, Figure S4 a). The qualitative observation was also performed in a confocal fluorescence microscope (with an inverted configuration) using negative and positive tracers with fluorescence emission in the green region. For the fluorescent negative tracers $\zeta=-31 \pm 2 \mathrm{mV}$ and $\mathrm{pH}=6.7$ whereas for the positive tracers $\zeta=56 \pm 2 \mathrm{mV}$ and $\mathrm{pH}=6.3$. In the case of the confocal fluorescence microscope, the sample was placed perpendicularly to the objective as schematized in Fig. S4 b of the supplementary data. 
For a quantitative determination of EZ, a Z-cut procedure (a z-stacking or focus stacking) was followed in unpatterned Nafion films. The inspection was performed with an inverted optical microscope in a setup with Nafion on top and the objective below. The z-stack process consisted of acquiring multiple images taken at different focal planes. The z-stack was generated by fast acquisition of images from the Nafion surface towards the bulk colloidal dispersion. The typical separation among focal planes was set to either 5 or $10 \mu \mathrm{m}$. The colloidal particles being excluded from the Nafion interface begin to move away delimiting a front of particles that can be captured by the z-stack procedure. The z-stack procedure was performed every 2 or 3 minutes to extract the evolution of EZ formation with time. At each time, we analyzed the images in the $\mathrm{z}-$ stack, and the location of the EZ is just given by the distance from the surface to the focal plane at which the front of particles becomes visible and in focus. The EZ quantification was achieved by evaluating 5 Nafion sample replicates for each kind of tracers.

Since the procedure was taken place with the Nafion sample on top and the objectives below, the particle tracers were also subjected to the gravity force. In order to subtract gravity effects, the same z-stack procedure was performed in a reference bare gold substrate (without Nafion), where only gravity effects were expected (see Fig. S5). The z-stack procedure was also repeated every 2 or 3 minutes to extract the evolution of the front of particle position with time and to calculate the particle sedimentation. The sedimentation velocity was evaluated experimentally with the same particle density $(0.2 \mathrm{mg} / \mathrm{ml})$ and salt concentrations $\left(1.24 \times 10^{-6} \mathrm{M}\right)$ than the ones used for characterizing the EZ. The actual properties of the EZ were obtained by subtracting the average sedimentation velocity of the tracer particles, measured on the Au substrate, from the velocity values obtained with the Nafion substrate. The velocities of the differently charged tracer particles as a function of the distance to the Nafion interface were obtained by first fitting 
the data of EZ vs. time to a smooth exponential interpolating function ${ }^{50}$ and then taking the derivative with respect to time and subtracting the average sedimentation velocity. The velocity vs. time and the EZ vs. time were then plotted parametrically to yield the plots of velocity $v s$. distance from the Nafion surface.

\subsection{Simulations}

In order to understand the main parameters controlling the EZ formation, finite element simulations using the software COMSOL Multiphysics v4.3 were implemented. The studies were performed by solving the stationary coupled governing equations:

$$
\begin{aligned}
& -\varepsilon \nabla^{2} \varphi=\rho_{e} \\
& \nabla \cdot \boldsymbol{v}=0 \\
& \eta \nabla^{2} \boldsymbol{v}=\nabla p+\rho_{e} \nabla \varphi \\
& \boldsymbol{v} \cdot \nabla C_{i}=\nabla \cdot\left(D_{i} \nabla C_{i}+z_{i} F \mu_{i} \nabla \varphi C_{i}\right) .
\end{aligned}
$$

Equation 1 stands for Poisson's equation for electrostatics, relating the local charge density $\rho_{e}=\sum_{i} F Z_{i} C_{i}$ with the electrostatic potential $\varphi$, where $\varepsilon\left(=\varepsilon_{r} \varepsilon_{0}\right)$ represents the permittivity of the liquid, $F$ is the Faraday's constant and $C_{i}$ and $z_{i}$ are the molar concentration and the valence of each ionic species, respectively, denoted by the subscript $i$. Equations 2 and 3 are the stationary Stokes' equations describing the motion of an incompressible fluid at low Reynolds numbers, where $\boldsymbol{v}, p$ and $\eta$ are the fluid velocity, pressure and viscosity, respectively. Finally, Eq. 4 is the stationary Nernst-Planck's equation for mass transport, where $D_{i}$ is the diffusion coefficient, and $\mu_{i}$ the mobility of the different species. The mobility is connected to the diffusion 
coefficient through Einstein's relation, $D_{i}=\mu_{i} R T$, where $R$ is the ideal gas constant and $T$ is the absolute temperature.

To avoid charge accumulation and to maintain steady-state conditions, we imposed in the simulations a local current exchange constraint at the Nafion interface, $j_{H^{+}}=-j_{\mathrm{M}^{+}}$, i.e., the rate of protons released by the Nafion into the solution has to be the same as the rate of uptaken salt cation $\left(\mathrm{M}^{+}\right)$. This requirement is implemented in the COMSOL simulations through the boundary conditions at the Nafion surface. Water equilibrium dissociation reaction is considered in the bulk liquid ${ }^{51}$.

Given the symmetry of the problem, a $2 \mathrm{D}$ section of the real system was implemented as simulation domain, which was discretized using an extremely fine mesh with 68600 triangular elements that was refined using boundary layers near the surface. A mesh density convergence study was performed to guarantee that discretization errors in the numerical solution were below $5 \%$ in all cases. Only stationary solutions were evaluated corresponding to steady state conditions. In the simulations, four different charged species were considered: protons, hydroxide ions, and different monovalent (1:1) salts. For the simulations with $\mathrm{C}_{\mathrm{d}}=1.24 \times 10^{-}$ ${ }^{3} \mathrm{~mol} / \mathrm{m}^{3}, \mathrm{NaCl}$ was used as salt.

The following boundary conditions were defined: $\varphi(\mathrm{z}=\mathrm{h})=0$ and $\varphi(\mathrm{z}=0)=\zeta_{\text {Nafion, }}$, where $h=$ $900 \mu \mathrm{m}$ is the height of the experimental cell and $\zeta_{\text {Nafion }}$ is the zeta potential of Nafion $\left(\zeta_{\text {Nafion }}=-\right.$ $0.034 \pm 0.002 \mathrm{~V}$ measured by dynamic light scattering with Nafion nanoparticles). For the fluid velocity, stick boundary conditions were imposed on the substrate and slip elsewhere. For the concentrations of the different species, the bulk values were imposed at the upper boundary $h$. The proper boundary conditions to be set for ion exchange at the Nafion interface are not well 
known. We performed simulations using two different sets of boundary conditions: by assuming that Nafion is a perfect drain for the salt cation, i.e., $c_{M^{+}}(z=0)=0$; or treating the ion exchange as a first order reaction characterized by a exchange rate $j_{M^{+}}(z=0)=-k_{e x} c_{M^{+}}(z=$ 0 ), where $k_{e x}$ is the exchange rate constant. In the analysis, the values of the electric field, and the proton concentration were evaluated as a function of the distance from the Nafion surface.

Table 1 collects the parameters used to make the simulations, as well as the dimensions of the 2D model detailed in the work.

\begin{tabular}{|c|c|c|}
\hline Parameter & Description & Value \\
\hline $\mathrm{D}_{\mathrm{H}}{ }^{+}$ & Diffusion coefficient of $\mathrm{H}^{+}$ & $9.3 \times 10^{-9} \mathrm{~m}^{2} / \mathrm{s}$ \\
\hline $\mathrm{D}_{\mathrm{OH}}{ }^{-}$ & Diffusion coefficient of $\mathrm{OH}^{-}$ & $5.3 \times 10^{-9} \mathrm{~m}^{2} / \mathrm{s}$ \\
\hline $\mathrm{D}_{\mathrm{Na}}{ }^{+}$ & Diffusion coefficient of $\mathrm{Na}^{+}$ & $1.33 \times 10^{-9} \mathrm{~m}^{2} / \mathrm{s}$ \\
\hline $\mathrm{D}_{\mathrm{K}}{ }^{+}$ & Diffusion coefficient of $\mathrm{K}^{+}$ & $1.96 \times 10^{-9} \mathrm{~m}^{2} / \mathrm{s}$ \\
\hline $\mathrm{D}_{\mathrm{Li}}{ }^{+}$ & Diffusion coefficient of $\mathrm{Li}^{+}$ & $1.03 \times 10^{-9} \mathrm{~m}^{2} / \mathrm{s}$ \\
\hline $\mathrm{D}_{\mathrm{Cs}}{ }^{+}$ & Diffusion coefficient of $\mathrm{Cs}^{+}$ & $2.06 \times 10^{-9} \mathrm{~m}^{2} / \mathrm{s}$ \\
\hline $\mathrm{D}_{\mathrm{Cl}}{ }^{-}$ & Diffusion coefficient of $\mathrm{Cl}^{-}$ & $1.0 \times 10^{-9} \mathrm{~m}^{2} / \mathrm{s}$ \\
\hline $\mathrm{T}^{\mathrm{T}}$ & Temperature & $298 \mathrm{~K}$ \\
\hline $\mathrm{W}_{\mathrm{Naf}}$ & Width of Nafion & $250 \mu \mathrm{m}$ \\
\hline $\mathrm{C}_{\mathrm{d}}$ & Bulk salt concentration & $1.24 \times 10^{-3} \mathrm{~mol} / \mathrm{m}^{3}$ \\
\hline $\mathrm{C}_{\mathrm{b}}$ & Water pH (bulk) & 6.6 \\
\hline$\zeta_{\mathrm{Nafion}}$ & Zeta potential Nafion & $-34 \mathrm{mV}$ \\
\hline $\mathrm{H}$ & Cell height & $900 \mu \mathrm{m}$ \\
\hline$\varepsilon_{\mathrm{r}}$ & Relative Permittivity & 78 \\
\hline & & \\
\hline & & \\
\hline & & \\
\hline & & \\
\hline
\end{tabular}




\begin{tabular}{|c|c|c|}
\hline $\mathrm{P}$ & Density & $10^{3} \mathrm{~kg} / \mathrm{m}^{3}$ \\
\hline$\eta$ & Dynamic Viscosity & $10^{-3} \mathrm{~Pa} \cdot \mathrm{s}$ \\
\hline
\end{tabular}

Table 1. Reference parameters used in the simulations

\section{Results and Discussion}

The EZ was characterized qualitatively by evaluating the interaction of positive and negative tracers with thin Nafion films supported on $\mathrm{Au} / \mathrm{Si}$ substrates (see Experimental Section). Two different Nafion configurations were explored: plain Nafion films or Nafion films with deactivated strips patterned with electron beam lithography. In the case of the strip-patterned Nafion, an EZ is readily built-up at the Nafion regions which were not exposed to the electron beam when the sample is immersed in a colloidal dispersion of negative particles. The colloidal tracers are confined in the deactivated channel regions, as depicted in Fig. 1a. The colloidal particle region in the channel becomes narrower with time (Fig. 1b) due to lateral EZ formation which pushes the tracers towards the central part of the channel. A scheme of the distribution of colloids is illustrated in Fig. 1c. We have also used a confocal fluorescence microscope to follow qualitatively the EZ formation. In that case, we placed a Nafion film supported on Au wafer perpendicularly to the microscope objective and immersed it in a negatively charged and fluorescent colloidal dispersion. Figure 1d depicts the fluorescence image of the sample showing an $\mathrm{EZ}$ of about $250 \mu \mathrm{m}$. 

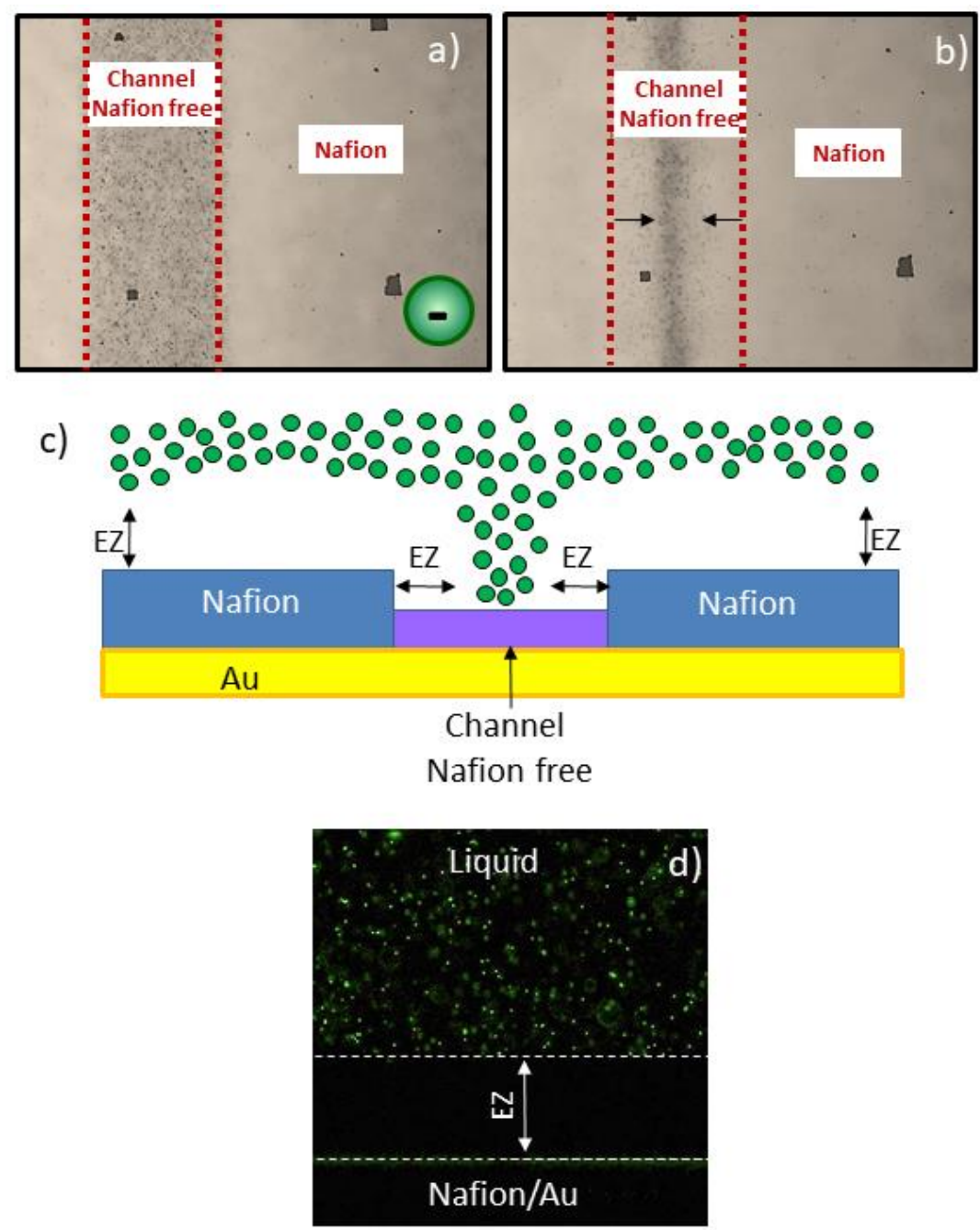

Figure 1. (a) EZ formation at the Nafion surface, characterized by the absence of tracer particles. The colloidal particles are only confined in the strip regions (Nafion-free channel) in which Nafion functionalities have been deactivated with electron beam lithography. (b) The particle region at the channel becomes narrower and more compressed with time. The optical inspection was performed with the Nafion sample on top and the objectives below (Fig. S4 a). (c) Schematics illustrating the EZ formation at the strip-patterned Nafion surface. (d) Confocal fluorescence image of EZ with fluorescent negative particles. In this case, the surface of the Nafion was placed perpendicularly to the objective (Fig. S4 b). 
It is important to emphasize that no EZ formation was observed when both kinds of Nafion samples were immersed in a colloidal dispersion of positive tracers. Figure 2 shows the absence of EZ either for the strip-patterned thin Nafion films or for the plain thin films placed in a perpendicular configuration. In the first case (Figs. $2 a$ and $2 b$ ) the particles are distributed over the entire surface, on both the Nafion regions and the deactivated channels. In the image of the Nafion thin film placed perpendicularly to the objective (Fig. 2c), the colloids distribute uniformly throughout the entire aqueous solution. The figure also shows an accumulation of fluorescent tracers just at the interface.

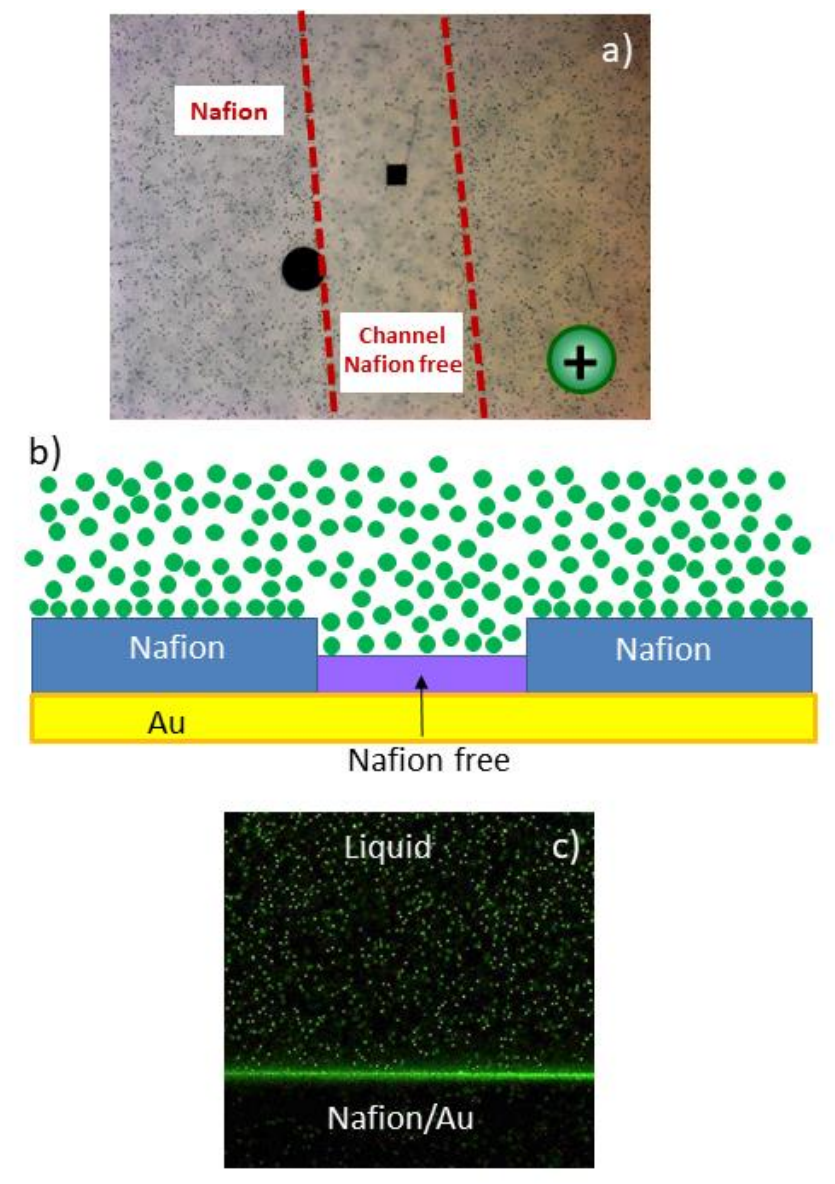


Figure 2. (a) Absence of EZ formation in the strip-patterned Nafion samples both on top of the Nafion surface and at the Nafion deactivated strips (Nafion-free channel). The optical inspection was performed with the Nafion sample on top and the objectives below (Fig. S4 a). (b) Schematics illustrating the absence of EZ formation at the entire strip-patterned Nafion surface. (c) Confocal fluorescence image of fluorescent positive particles which are distributed in the bulk liquid and accumulated at the Nafion interface. The image was taken placing the Nafion sample perpendicularly to the microscope objective (Fig. S4 b).

A quantitative analysis of the EZ formation was performed by calculating the particle displacement with a z-stack procedure as detailed in the Experimental section. Figure 3a shows the time dependence of the EZ formation after subtracting gravity effects. The exclusion distances progress faster at shorter times reaching a plateau at longer times.

We have explored the effect of salt on the EZ formation by externally adding different concentrations of $\mathrm{NaCl}$ to the negatively charged colloidal dispersion (Fig. 3b). Although the time evolution trend of the EZ formation is similar for the different salt concentrations, a drop of the exclusion distances is observed as the concentration of salt increases. Remarkably, the EZ persists even at relatively high $\mathrm{mM}$ salt concentrations. Another interesting result is that the EZ is sensitive to the diffusion coefficient of the salt. Figure $3 \mathrm{c}$ shows the evolution of the EZ length in the presence of $\mathrm{LiCl}$ and $\mathrm{CsCl}$ salts, respectively, with diffusion coefficients of $1.03 \times 10^{-9} \mathrm{~m}^{2} \mathrm{~s}^{-}$ ${ }^{1}$ for $\mathrm{Li}^{+}$and $2.06 \times 10^{-9} \mathrm{~m}^{2} \mathrm{~s}^{-1}$ for $\mathrm{Cs}^{+}$. Larger EZs were observed in presence of lithium salts. 

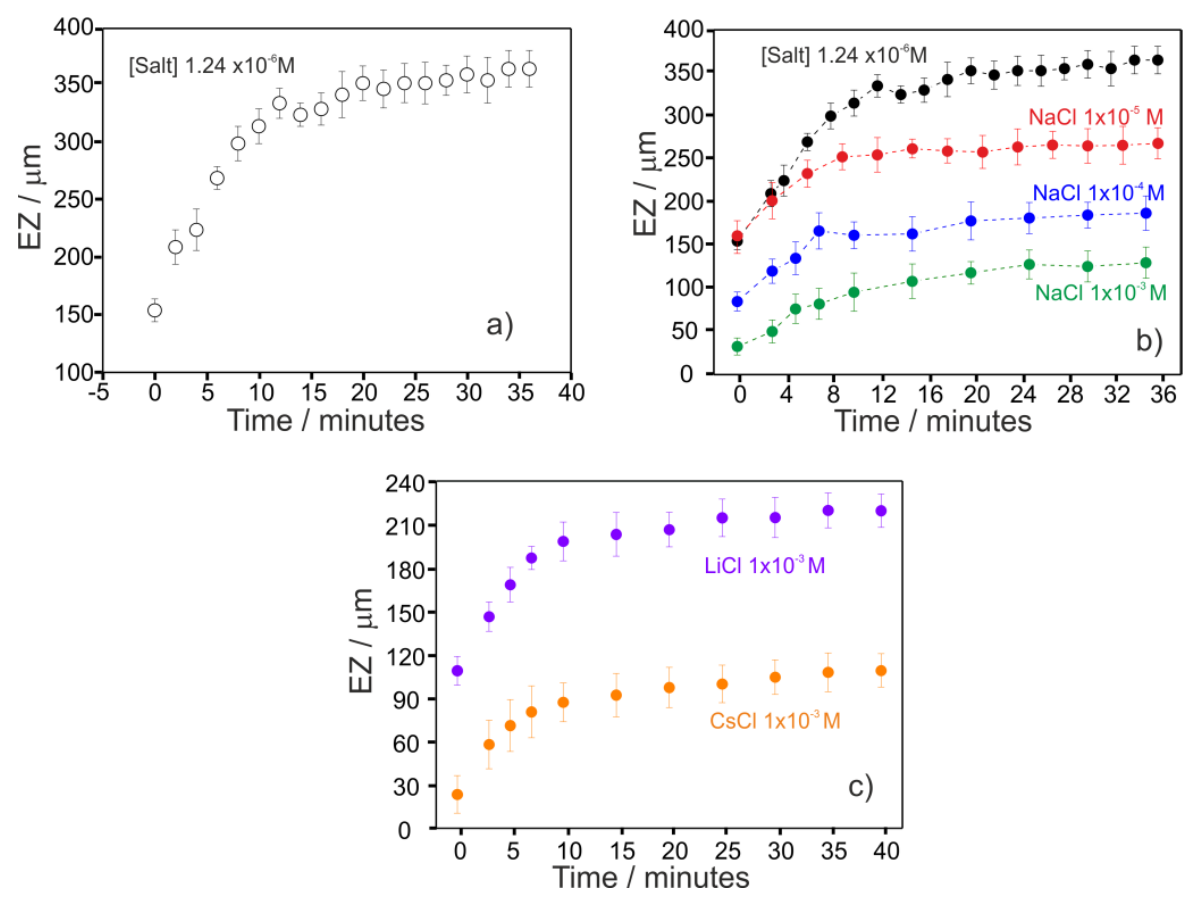

Figure 3. (a) Length of the EZ as a function of time in presence of negatively charged particles without external addition of salt. The residual concentration of salt in such colloidal dispersion was estimated to be $1.24 \mu \mathrm{M}$ by performing calibration curves of conductimetry. (b) Time evolution of the EZ length for different concentration of externally added salts. (c) EZ evolution in presence of negative particles with external addition of salts with different diffusion coefficients. In all cases, the effects of gravity have been subtracted.

The EZ sensitivity to solute charge, salt concentration and diffusion coefficients suggests the presence of an interfacial electric field driving the formation of the EZ. In order to evaluate and quantify the potential presence of an electric field near the Nafion/liquid interface, we characterized the EZ evolution by using negative tracers with different zeta potentials. Specifically, we used carboxylated PS particles $(\zeta=-0.042 \pm 0.003 \mathrm{~V})$ and weakly charged plain PS particles $(\zeta=-0.012 \pm 0.001 \mathrm{~V})$. Figure $4 \mathrm{a}$ shows the time evolution of the EZ at the Nafion 
interface for the two differently charged negative tracers without subtracting gravity effects. As expected, larger EZs are achieved with the more negatively charged particles. From these plots, the particle velocities drifting away from the Nafion interface can be obtained as explained in the Experimental part. Figure $4 \mathrm{~b}$ shows the velocity of the colloidal particles already corrected by gravity and extrapolated up to the Nafion interface. The figure shows that the carboxylated particles move away from the Nafion interface at higher velocities than the weakly charged PS particles. This suggests the existence of an electric field. This electric field arises from the Nafion capability of exchange protons by other cations of different diffusion coefficients, giving rise to concentration gradients that trigger ionic diffusiophoretic forces acting on solute particles. In a multi-ion solution the diffusiophoretic velocity of particles with low $\zeta$ values and in the Helmholtz-Smoluchowski limit $(\mathrm{r} / \lambda>>1)$ can be expressed as ${ }^{52,51}$ :

$$
v_{d p}=\frac{\varepsilon}{8 \eta} \frac{\sum_{i} z_{i}^{2} \nabla n_{i}}{\sum_{i} z_{i}^{2} n_{i}} \zeta_{p}^{2}+\frac{\varepsilon E}{\eta} \zeta_{p}
$$

where

$$
E=\frac{k_{B} T}{e} \frac{\sum_{i} z_{i} D_{i} \nabla n_{i}}{\sum_{i} z_{i}^{2} D_{i} n_{i}}
$$

is the electric field generated by the different diffusivity $D_{i}$ of the ions, $n_{i}$ is the number concentration of ion type $i, \zeta_{p}$ is the zeta potential of the particle and $\varepsilon$ and $\eta$ stand for the permittivity and the viscosity of the fluid, respectively. $e$ and $k_{B}$ represent the proton charge and the Boltzmann's constant, respectively. 
According to Eq. 5, the velocity of the solute particles due to multi-ionic diffusiophoresis has two contributions: a chemiphoretic contribution (first term) proportional to $\zeta_{p}^{2}$ and an electrophoretic one (second term) proportional to the electric field $E$ and to $\zeta_{p}$. The chemiphoretic contribution arises as a consequence of the non-uniform pressure built-up on the particle double layer due to the salt concentration gradient. This contribution always induces fluid flow toward lower electrolyte concentration, generating particle motion in the opposite direction to balance such effect ${ }^{21}$. Instead, the electrophoretic contribution arises from a local electric field created by the difference of ion diffusivities, and its direction depends on the sign of $\zeta_{p}$ and the sign of the electric field. We can rewrite the velocity of solute particles due to multi-ionic diffusiophoresis as:

$$
v_{d p}=a \zeta_{p}^{2}+\frac{\varepsilon E}{\eta} \zeta_{p}
$$

The velocities of two differently-charged particles $\left(v_{p}\right.$ - and $\left.v_{p 0}\right)$ will then be given by:

$$
\begin{aligned}
& v_{p-}=a \zeta_{p-}^{2}+\frac{\varepsilon E}{\eta} \zeta_{p-} \\
& v_{p 0}=a \zeta_{p 0}^{2}+\frac{\varepsilon E}{\eta} \zeta_{p 0} .
\end{aligned}
$$

Combining these two equations, we can extract the normal component of the electric field as:

$$
E=\frac{\eta}{\varepsilon} \frac{\left(v_{p_{0}} \zeta_{p_{-}}^{2}-v_{p_{-}} \zeta_{p_{0}}^{2}\right)}{\left(\zeta_{p_{0}} \zeta_{p_{-}}^{2}-\zeta_{p_{-}} \zeta_{p_{0}}^{2}\right)}
$$

where $v_{p_{-}}$and $\zeta_{p_{-}}$and $v_{p_{0}}$ and $\zeta_{p_{0}}$ are the normal velocities and zeta potentials for the carboxylated PS $(-0.042 \mathrm{~V})$ and plain PS $(-0.012 \mathrm{~V})$ particles, respectively. Therefore, from the 
experimental knowledge of the velocities of the two types of tracers at different distances, we can estimate the value of the normal component of the electric field as a function of the distance from the Nafion surface. Different approximations have been considered in the evaluation of the electric field. The experimental values of the zeta potentials are not strictly in the HelmholtzSmoluchowski limit, as mentioned in the experimental section, and it has also been assumed that they not change within the salt gradient and that they are relatively low. These approximations are used for consistency since a pure experimental analysis taking into account the concentration dependence of the zeta potential would have not been feasible without implementing a simultaneous technique to determine the actual ionic concentration gradient. The error associated to the use of these approximations is within the error bars of the estimated electric field.

Figure $4 \mathrm{c}$ shows the values of the component of the electric field normal to the Nafion surface as a function of the distance from the Nafion interface obtained from the experimental measurement of the velocities of the differently charged colloids and applying Eq. 10. The electric field decreases towards the bulk electrolyte and has an estimated maximum value of -42 $\mathrm{V} / \mathrm{m}$ at the outer region of the Nafion surface obtained by extrapolation. In addition, the individual strength of the electrophoretic and chemiphoretic contributions was also estimated from the experimental values of the velocities of colloidal tracers with different zeta potentials and the electric field and applying Eqs. 8 and 9. Figure 4d shows both contributions for the case of the weakly charged PS particles as a representative system. The figure clearly depicts that the electrophoretic contribution dictates the particle velocity which becomes stronger as the distance towards the Nafion surface decreases. The chemiphoretic velocity is very small and only becomes comparable to the electrophoretic one at very long distances from the Nafion interface, when both contributions are almost negligible. 

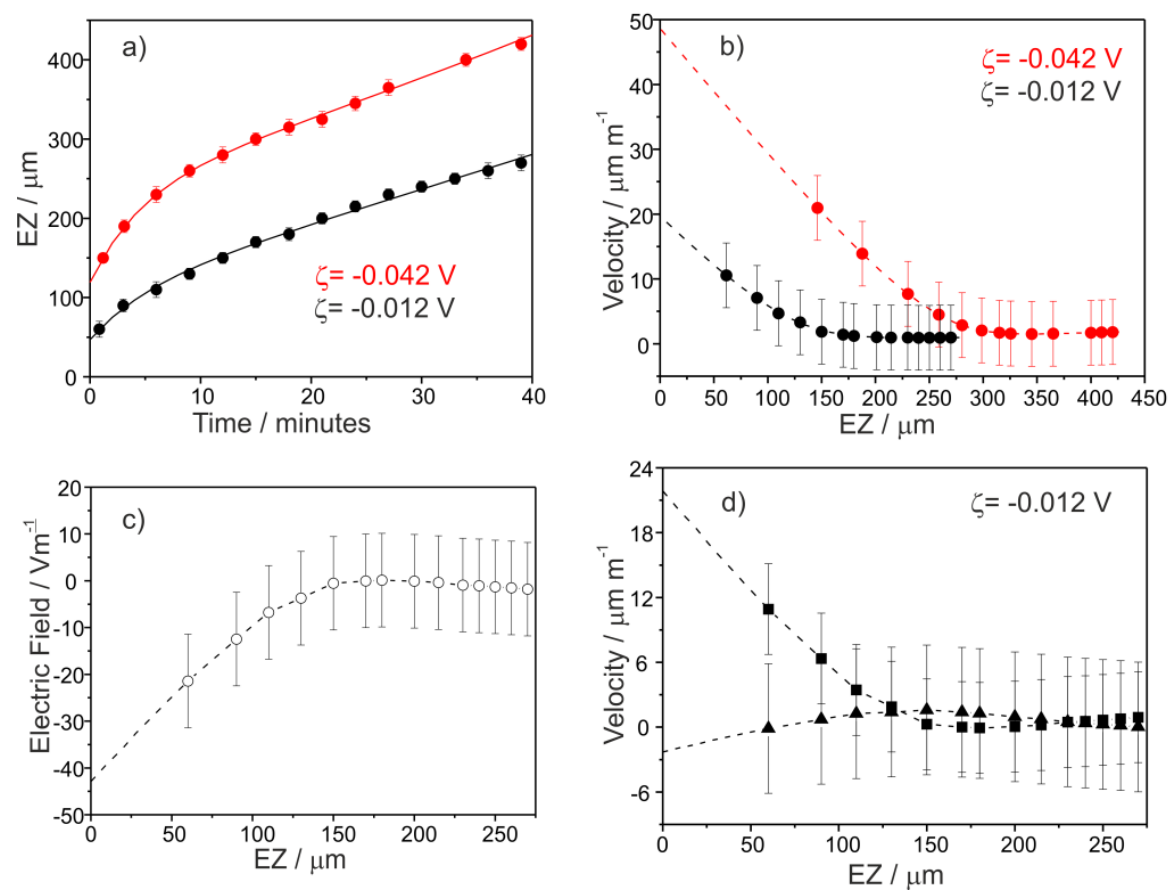

Figure 4. (a) EZ evolution as a function of time for highly and weakly charged negative tracers. The dots represent the experimental data and the lines are the fits performed to evaluate the velocity (see Experimental section). (b) Velocity profiles for the highly and weakly charged negative tracers as a function of the distance from the Nafion surface. (c) Electric field as a function of the distance from the Nafion surface evaluated using Eq. 10. (d) Comparison between the electrophoretic (full squares) and chemiphoretic (full triangles) contributions to the particle velocity as a function of the distance from the Nafion surface for the weakly-charged negative tracers. The electric field and the different phoretic contributions were obtained from the experimental measurement of the velocities of differently charged colloidal tracers.

In order to better understand these experimental results, numerical simulations (COMSOL Multiphysics) were done by solving the governing equations involved in this complex and coupled ion exchange/diffusion/migration/electrostatic problem. Figure 5a shows a cross-section 
of the 2D layout geometry used for the simulations: a Nafion film of $250 \mu \mathrm{m}$ length in contact with an electrolyte layer of $900 \mu \mathrm{m}$ in height. The solution of the governing equations clearly shows the presence of a perpendicular electric field near the polymer/liquid interface, pointing towards the Nafion surface (Figure 5a). The electric field extends far beyond the double layer correlating well with the observed exclusion zones reaching hundreds of microns. The existence and direction of the electric field agree well with the experimental findings in which formation and absence of EZ were observed in presence of negative and positive tracers, respectively. The direction of such field would repel negatively charged particles and attract the positive ones.

Figure $5 \mathrm{~b}$ depicts the value of the electric field as a function of the distance from the Nafion obtained directly from the simulations. We have compared this electric field with the theoretical prediction, Eq. (6), evaluated using the diffusion coefficients and the concentration gradients of the four ions $\left(\mathrm{H}^{+}, \mathrm{OH}^{-}, \mathrm{Na}^{+}, \mathrm{Cl}^{-}\right)$resulting from the simulations. The quantitative values of the electric field obtained directly in the simulation agree remarkably well with the theoretical predictions of Eq. 6, as depicted in Fig 5b, confirming the multi-ionic diffusiophoresis scenario. Figure $5 \mathrm{c}$ shows the spatial distribution of the total ion concentration and the concentrations of protons and $\mathrm{Na}^{+}$ions. The Nafion polymer is initially protonated but in presence of salt cations $\left(\mathrm{Na}^{+}, \mathrm{K}^{+}\right.$, etc. $)$an ion exchange is produced inducing a spatial gradient of such ions at the interface. The proton concentration is higher near the Nafion interface, due to the proton release in exchange of the $\mathrm{Na}^{+}$captured from the solution, and then decreases until the bulk $\mathrm{pH}(\mathrm{pH}=6.6)$ is reached. However, the total concentration of ions is smaller near the Nafion interface, in agreement with previous studies ${ }^{11}$.

We have also simulated the effect of the diffusion coefficients of exchanged cation $\left(\mathrm{Li}^{+}, \mathrm{Na}^{+}\right.$, $\mathrm{K}^{+}$, and $\mathrm{Cs}^{+}$) (Figure 5d) on the generated electric field. The results of the simulations clearly 
indicate that the electric field generated near the interface of Nafion decreases by increasing the diffusion coefficient of the salt. In particular, the electric field is larger in the case of $\mathrm{LiCl}$ than in the case of $\mathrm{CsCl}$. These results correlate well with the experimental findings of Fig. $3 \mathrm{c}$ in which a larger EZ was found with negatively charged colloidal dispersions containing $\mathrm{LiCl}$ salts. The higher electric field generated at the interface when protons are exchanged by $\mathrm{Li}^{+}$ions, can be rooted in the higher difference of diffusion coefficient values between protons $\left(9.3 \times 10^{-9} \mathrm{~m}^{2} \mathrm{~s}^{-1}\right)$ and $\mathrm{Li}^{+}\left(1.03 \times 10^{-9} \mathrm{~m}^{2} \mathrm{~s}^{-1}\right)$ as compared with $\mathrm{Cs}^{+}\left(2.06 \times 10^{-9} \mathrm{~m}^{2} \mathrm{~s}^{-1}\right)$, and fulfills the behavior predicted by Eq. 6 .
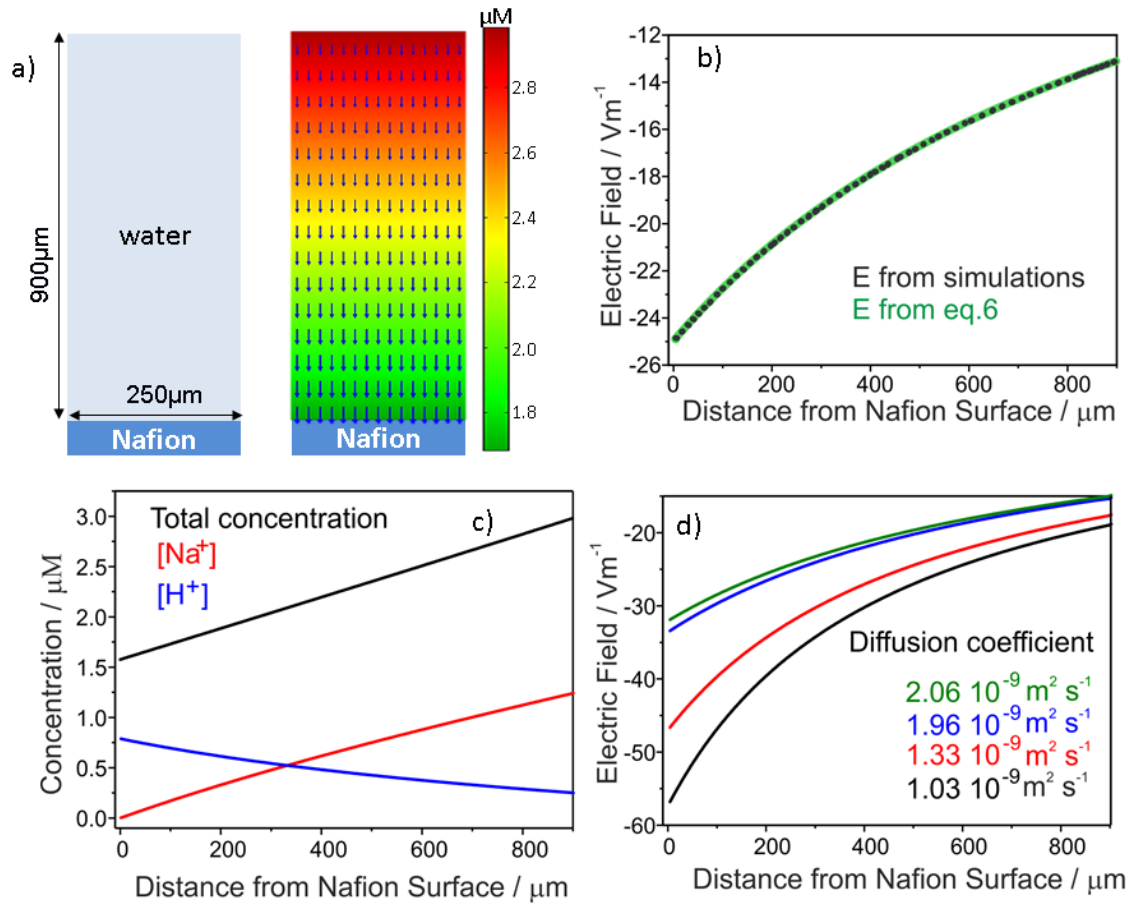

Figure 5. (a) Scheme of the model layout used for the simulations together with the $2 \mathrm{D}$ electric field (arrows) and 2D map of the total ion concentration at the Nafion/water interface (color scale). (b) Comparison of the value of the normal electric field as a function of the distance from the Nafion surface obtained from simulations (dotted curve) and calculated from Eq. 6 (solid curve). (c) Spatial variation of the sodium (red), proton (blue) and total (black) ion 
concentrations obtained in the simulations. (d) Spatial variation of the normal component of the electric field for a multi-ion diffusion process containing protons, oxyhydriles, chlorides and cations of different diffusion coefficients, $\mathrm{Li}^{+}\left(1.03 \times 10^{-9} \mathrm{~m}^{2} / \mathrm{s}\right.$, black line), $\mathrm{Na}^{+}\left(1.33 \times 10^{-9} \mathrm{~m}^{2} / \mathrm{s}\right.$, red line), $\mathrm{K}^{+}\left(1.96 \times 10^{-9} \mathrm{~m}^{2} / \mathrm{s}\right.$, blue line $), \mathrm{Cs}^{+}\left(2.06 \times 10^{-9} \mathrm{~m}^{2} / \mathrm{s}\right.$, green line). The simulations were evaluated for salt concentrations of alkali metal chloride of $1 \mathrm{mM}$. All the simulations were performed in quasi steady state conditions.

Finally, we have also analyzed by simulation the behavior of the electric field as a function of salt concentration (Figs. S6a and b). The results depend on the ion exchange mechanism (which in the simulations is implemented by a different boundary condition). If we assume that the Nafion acts as a perfect sink of positively charged ions, the electric field increases monotonically with the addition of salt up to reaching a limiting value. Contrarily, if we assume that the ionexchange is a first-order reaction with an exchange rate constant $k_{e x}$, the field first increases with salt concentration, reaches a maximum, and then decreases at higher concentrations. The salt concentration at which the electric fields starts to decrease depends on the value of $k_{e x}$, becoming smaller for small values of $k_{e x}$. A slow decrease in the electric field upon the addition of salt is the behavior found experimentally, suggesting that the ion exchange at high concentrations is somehow reaction-limited rather than diffusion limited. At low salt concentration and if the exchange rate constant is large enough, both boundary conditions yield consistently the same results, suggesting that under those conditions, the ion exchange rate is diffusion limited. Remarkably, the experiments show that the electric field persists at $\mathrm{mM}$ salt concentrations. This is a relevant finding especially for potential applications which operate in moderate saline media. 
Our experimental and theoretical results confirm the diffusiophoretic origin of the mechanism of EZ formation stemming from the natural capabilities of Nafion as ion exchanger, as suggested by Wyss and coworkers. The exchange of ions with different diffusion coefficients induces ion gradients and ion diffusion with the consequent build-up of an electric field. An electric field directed towards the Nafion surface is established when protonated Nafion is immersed in a salt containing electrolyte. Therefore, solutes (e.g., colloidal tracers) will experience multi-ionic diffusiophoresis in such ionic gradient which will make them move against or towards the Nafion surface. The balance of the electrophoretic and chemiphoretic contributions will dictate the colloid velocity and direction ${ }^{53}$. We have shown that the electrophoretic contribution prevails over the chemiphoretic one, playing the key role in EZ formation. Chemiphoretic effects can only exert a modest impact at longer distances $(>200 \mu \mathrm{m})$ from the Nafion interface when the electric field becomes negligible. The relevant role of the electrophoretic force also explains the modulation of EZ with the $\zeta$ of the solute, the salt concentration and the cation diffusion coefficients. Thus, not all solutes can be expelled from the Nafion surface and form EZs. As $\zeta$ become less negative the EZ decreases and eventually disappears completely for positively charged solutes, which become attracted and accumulate at the surface. The effect of salt concentration on the electric field and EZ exhibits a more complex dependence because it is influenced by the kinetics of the ion exchange mechanism. A decrease in the electric field with salt concentration takes place when the ion exchange becomes reaction limited. Finally, stronger electric fields and higher EZ can be reached if the difference between the ion diffusion coefficients of the exchanged ions is larger. 


\section{Conclusions}

In summary, we have evaluated the mechanism of formation of long-range solute-free regions generated near thin Nafion films in contact with alkali metal halide aqueous solutions. The EZ formation is well explained considering that solutes are subjected to multi-ionic diffusiophoresis near the Nafion/water interface, as indicated by Florea et al. We have evaluated the selfgenerated electric field, extending well beyond the double layer and demonstrated its key role in multi-ionic diffusiophoresis. Therefore, the EZ formation does not stem from water structuring effects at the Nafion interface. Neither is it dominantly driven by the chemiphoretic contribution of ionic diffusiophoresis, at least in the presence of alkali metal chlorides. The extent of the EZ can be modulated by changing the electric field strength with ions of different diffusion coefficients, by changing salt concentration, or with solutes of different surface charge.

Our work provides a more detailed explanation of the striking phenomenon of large EZs in ion-exchange polymers as Nafion. A precise knowledge of ion gradients produced by the ion exchange, with the consequent generation of electric fields at Nafion, can be used to boost novel applications such as unique micro/nanofluidic devices with patterned Nafion or Nafion based self-propelled micro/nanomotors.

\section{Supporting Information.}

Details on i) the electron beam patterning of the Nafion film, ii) contact angle and ii) ion exchange capacity measurements of the thin Nafion films, iv) schemes of the optical measurement set-up, v) sedimentation velocities of the differently charged particle tracers and vi) the effect of salt concentration studied by numerical simulations. 


\section{Acknowledgment}

This research was supported by the Spanish Ministry of Economy and Competitiveness (MINECO) under Contract Nos. MAT2015-68307-P, FIS2015-67837, and PGC2018-095032-B100. The ICN2 is funded by the CERCA program/Generalitat de Catalunya. The ICN2 is supported by the Severo Ochoa program of MINECO (Grant SEV-2017-0706). We thank the

students R. Salavert, A. Sarria and Y. Fetisova for helping us in the sample preparation and microscopy measurements.

\section{References}

$1 \quad$ K. Green and T. Otori, J. Physiol., 1970, 207, 93-102.

2 B. V. Derjaguin and M. V. Golovanov, Prog. Surf. Sci., 1992, 40, 210-217.

3 B. Chai, A. G. Mahtani and G. H. Pollack, Contemp. Mater., 2012, 3, 1-12.

4 J. ming Zheng and G. H. Pollack, Phys. Rev. E - Stat. Physics, Plasmas, Fluids, Relat. Interdiscip. Top., 2003, 68, 031408.

5 J. Zheng, A. Wexler and G. H. Pollack, J. Colloid Interface Sci., 2009, 332, 511-514.

$6 \quad$ K. A. Mauritz and R. B. Moore, Chem. Rev., 2004, 104, 4535-4586.

7 A. Kusoglu and A. Z. Weber, Chem. Rev., 2017, 117, 987-1104.

8 B. Domènech, M. Muñoz, D. N. Muraviev and J. Macanás, Chem. Commun., 2014, 50, 4693-4695.

9 B. Chai, H. Yoo and G. H. Pollack, J. Phys. Chem. B, 2009, 113, 13953-13958.

10 R. Das and G. H. Pollack, Langmuir, 2013, 29, 2651-2658.

11 D. Florea, S. Musa, J. M. R. Huyghe and H. M. Wyss, Proc. Natl. Acad. Sci., 2014, 111, $6554-6559$.

12 C. O’Rourke, I. Klyuzhin, J. S. Park and G. H. Pollack, Phys. Rev. E, 2011, 83, 056305. 
13 X. A. Figueroa and G. H. Pollack, Int. J. Des. Nat. Ecodynamics, 2011, 6, 286-296.

14 S. A. Skopinov, M. V. Bodrova, M. P. R. Jablon, G. H. Pollack and F. A. Blyakhman, J. Solution Chem., 2017, 46, 626-632.

15 S. G. Hwang, J. K. Hong, A. Sharma, G. H. Pollack and G. W. Bahng, PLoS One, 2018, 13, $1-17$.

16 L. Cheng, P. Fenter, K. L. Nagy, M. L. Schlegel and N. C. Sturchio, Phys. Rev. Lett., 2001, 87, 156103.

17 T. Fukuma, Y. Ueda, S. Yoshioka and H. Asakawa, Phys. Rev. Lett., 2010, 104, 016101.

18 J. M. Schurr, J. Phys. Chem. B, 2013, 117, 7653-7674.

19 J. M. Schurr, B. S. Fujimoto, L. Huynh and D. T. Chiu, J. Phys. Chem. B, 2013, 117, $7626-7652$.

20 S. Musa, D. Florea, H. M. Wyss and J. M. Huyghe, Soft Matter, 2016, 12, 1127-1132.

21 J. Anderson, Annu. Rev. Fluid Mech., 1989, 21, 61-99.

22 M. M. J. Lin and D. C. Prieve, J. Colloid Interface Sci., 1983, 95, 327-339.

23 W. J. Lechnick and J. A. Shaeiwitz, J. Colloid Interface Sci., 1984, 102, 71-87.

24 J. P. Ebel, J. L. Anderson and D. C. Prieve, Langmuir, 1988, 4, 396-406.

25 P. O. Staffeld and J. A. Quinn, J. Colloid Interface Sci., 1989, 130, 69-87.

26 B. Abécassis, C. Cottin-Bizonne, C. Ybert, A. Ajdari and L. Bocquet, Nat. Mater., 2008, 7, 785-789.

27 J. Palacci, C. Cottin-Bizonne, C. Ybert and L. Bocquet, Soft Matter, 2012, 8, 980-994.

28 J. Palacci, B. Abécassis, C. Cottin-Bizonne, C. Ybert and L. Bocquet, Phys. Rev. Lett., 2010, 104, 138302.

29 J. Diao, L. Young, S. Kim, E. A. Fogarty, S. M. Heilman, P. Zhou, M. L. Shuler, M. Wu 
and M. P. DeLisa, Lab Chip, 2006, 6, 381-388.

30 R. Niu, E. C. Oğuz, H. Müller, A. Reinmüller, D. Botin, H. Löwen and T. Palberg, Phys. Chem. Chem. Phys., 2017, 19, 3104-3114.

31 B. Liebchen, R. Niu, T. Palberg and H. Löwen, Phys. Rev. E, 2018, 98, 052610.

32 T. Okada, S. Møller-Holst, O. Gorseth and S. Kjelstrup, J. Electroanal. Chem., 1998, 442, $137-145$.

33 H. Zhang and P. K. Shen, Chem. Rev., 2012, 112, 2780-2832.

34 J. Wang, M. Musameh and Y. Lin, J. Am. Chem. Soc., 2003, 125, 2408-2409.

35 Y. Fan, J.-H. Liu, H.-T. Lu and Q. Zhang, Colloids Surfaces B Biointerfaces, 2011, 85, 289-292.

36 Y. Cheng and C. I. Moraru, Colloids Surfaces B Biointerfaces, 2018, 162, 16-24.

37 S. Shin, P. B. Warren and H. A. Stone, Phys. Rev. Appl., 2018, 9, 34012.

38 C. S. Chen, E. Farr, J. M. Anaya, E. Y. T. Chen and W. C. Chin, Entropy, 2015, 17, 14661476.

39 H. Lee, J. Kim, J. Yang, S. W. Seo and S. J. Kim, Lab Chip, 2018, 18, 1713-1724.

40 S. Ebbens, D. A. Gregory, G. Dunderdale, J. R. Howse, Y. Ibrahim, T. B. Liverpool and R. Golestanian, EPL (Europhysics Lett., 2014, 106, 58003.

41 A. Reinmüller, E. C. Oğuz, R. Messina, H. Löwen, H. J. Schöpe and T. Palberg, J. Chem. Phys., 2012, 136, 164505.

42 A. Reinmüller, H. J. Schöpe and T. Palberg, Langmuir, 2013, 29, 1738-1742.

43 R. Niu, P. Kreissl, A. T. Brown, G. Rempfer, D. Botin, C. Holm, T. Palberg and J. De Graaf, Soft Matter, 2017, 13, 1505-1518.

44 M. J. Esplandiu, A. Afshar Farniya and A. Bachtold, ACS Nano, 2015, 9, 11234-11240. 
45 K. Zhang, J. Fraxedas, B. Sepulveda and M. J. Esplandiu, ACS Appl. Mater. Interfaces, $2017,9,44948-44953$.

46 M. N. Popescu, W. E. Uspal and S. Dietrich, J. Phys. Condens. Matter, 2017, 29, 134001.

47 M. J. Esplandiu, K. Zhang, J. Fraxedas, B. Sepulveda and D. Reguera, Acc. Chem. Res., 2018, 51, 1921-1930.

48 A. Donev, A. J. Nonaka, C. Kim, A. L. Garcia and J. B. Bell, Phys. Rev. Fluids, 2019, 4, 043701.

49 A. Gupta, S. Shim, L. Issah, C. McKenzie and H. A. Stone, Soft Matter, 2019, 15, $9965-$ 9973.

50 D. Reguera, P. K. Rawlings and G. Birnbaum, J. Chem. Phys., 2013, 138, 224109.

51 A. Brown and W. Poon, Soft Matter, 2014, 10, 4016-4027.

52 T. Y. Chiang and D. Velegol, J. Colloid Interface Sci., 2014, 424, 120-123.

53 H. J. Keh and H. C. Ma, Langmuir, 2005, 21, 5461-5467. 

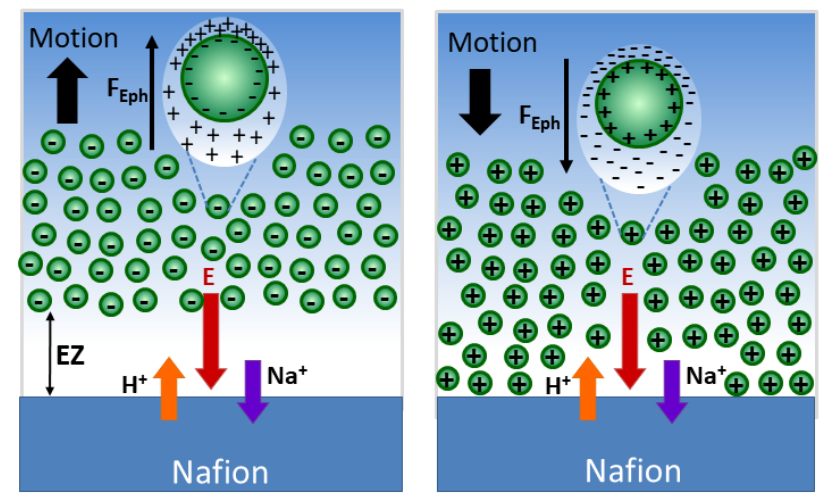

The Nafion exclusion zone formation has been investigated by evaluating experimentally and with simulations the electrophoretic and chemiphoretic contributions of multi-ionic diffusiophoresis. 